\title{
Biventricular myocardial function in Covid-19 recovered patients assessed by speckle tracking echocardiography: a prospective cohort echocardiography study
}

\author{
Elsi S. Tryfou ${ }^{1}$ (1) Peggy M. Kostakou ${ }^{2} \cdot$ Christos G. Chasikidis $^{1} \cdot$ Vassilios S. Kostopoulos $^{2} \cdot$ loannis I. Serafetinidis $^{1}$. \\ Efterpi K. Ferdianaki ${ }^{1} \cdot$ Constantinos Mihas ${ }^{3}$. Christoforos D. Olympios ${ }^{2} \cdot$ Nikos T. Kouris $^{2}$
}

Received: 29 September 2021 / Accepted: 14 December 2021 / Published online: 23 December 2021

(c) The Author(s), under exclusive licence to Springer Nature B.V. 2021

\begin{abstract}
Although severe acute respiratory syndrome coronavirus 2 causes respiratory disease, it may also lead to cardiovascular involvement with unknown long-term consequences. The aim of our study was to evaluate prospectively cardiac involvement in patients after the recovery from Covid-19, using two-dimensional speckle tracking echocardiography. 100 Covid-19 recovered patients with preserved left ventricular ejection fraction, were included, divided based upon clinical manifestation into two groups, those who were treated ambulant and those who were hospitalized. All patients underwent echocardiographic evaluation after their recovery. Although overall LV systolic function expressed by EF was normal, left ventricular global longitudinal strain (LVGLS) was significantly lower in Covid-19 recovered patients (33.28 \pm 9.4 days after diagnosis) compared to controls $(-18.47 \pm-2.4$ vs. $-21.07 \pm-1.76 \%$ respectively, $\mathrm{p}<0.0001)$. Especially the lateral wall longitudinal strain (LATLS) and posterior wall longitudinal strain (POSTLS) were significantly reduced in all patients compared to controls $(-17.77 \pm-3.48$ vs. $-20.97 \pm-2.86 \%, \mathrm{p}<0.0001$ and $-19.52 \pm-5.3$ vs. $-22.23 \pm-2.65 \%, \mathrm{p}=0.01)$. right ventricular global longitudinal strain (RVGLS) was significantly diminished only in the hospitalized group of Covid19 recovered patients, compared to controls $(-18.17 \pm-3.32$ vs. $-26.03 \pm-4.55 \%$ respectively, $p<0.0001)$. LVGLS is affected in almost all individuals after Covid-19 infection independently of the infection severity, with LATLS being the most sensitive marker of LV impairment and with POSTLS to follow. RV shows impaired GLS in severely ill patients highlighting RVGLS as a helpful tool of prognosis. Recovered patients from Covid-19 infection have to be monitored for a long time, since the term "long Covid disease" might also include the cardiac function.
\end{abstract}

Keywords Strain $\cdot$ Covid- $19 \cdot$ Speckle tracking echocardiography $\cdot$ Myocarditis

\section{Introduction}

Severe acute respiratory syndrome coronavirus 2 (SARSCoV-2) causes respiratory disease of variable severity and may lead to multi-system dysfunction. Although it primarily affects the lungs, cardiovascular complications such as

Elsi S. Tryfou

elsietr@gmail.com

1 Cardiology Department, General Hospital of Korinthos, Athinon 53, 20100 Corinth, Greece

2 Cardiology Department, General Hospital of Elefsina "Thriassio", Athens, Greece

3 Internal Medicine Department, General Hospital of Kymi, Evia, Greece myocarditis, arrhythmias and myocardial damage occur in $20-30 \%$ of hospitalized patients, resulting worse outcome [1, 2]. Especially viral myocarditis has been referred in multiple case reports and reviews and has been identified as a cause of death in Covid-19 patients [3]. On the other hand, high incidence of pre-existing cardiovascular disease has been noted among Covid-19 patients, followed by increased mortality [4]. In children infected with Covid-19, a hyperinflammatory condition has been observed, indicating characteristics similar to Kawasaki disease including heart and coronary vessel dysfunction [5]. Moreover, elevated high-sensitive cardiac troponin I (hs-cTnI) has been reported in 12-18\% of patients with Covid-19 during hospitalization, and cardiac involvement in these patients has been documented in $31 \%$ of severe-infected [6, 7]. All the previous data demonstrate a two-way process between Covid-19 and cardiovascular 
function, but the underlying mechanisms that elaborate this interaction remain unclear. It is, therefore, highly important to detect on time any early signs of cardiovascular involvement during and after Covid-19 infection. While vaccine programs are being accelerated in all over the world and clinical trials for effective anti-virus drugs are evolving, extended complications and late manifestations of Covid19 infection have been recognized, rising serious concern about possible "long Covid disease" [8]. The mechanism of cardiac damage remains poorly interpretable pointing out the management of these patients being particularly challenging.

Two-dimensional speckle tracking echocardiography (2D-STE) has the ability of early detecting subclinical myocardial dysfunction and has been used in many studies for the assessment of systolic function of left and right ventricle during the infection with Covid-19. Nevertheless, it is not clear whether cardiovascular involvement remains after Covid-19 infection recovery, since only a few studies with small cohort of patients have investigated long-term cardiac complications $[9,10]$.

The aim of our study was to evaluate prospectively myocardial systolic function and hence cardiac involvement in patients after the recovery from Covid-19, using 2D-STE.

\section{Materials and methods}

\section{Study population}

We performed a prospective cohort study, which took place from March 2021 until June 2021 at the General Hospital of Korinthos and at the General Hospital of Elefsina, 'Thriassio'. One hundred Covid-19 infected patients with preserved left ventricular ejection fraction (LVEF) and without cardiovascular involvement were enrolled. Based upon clinical manifestation of the disease, they were divided in two groups: the first group was consisted of 67 patients who were hospitalized due to pneumonia and respiratory insufficiency and the second was consisted of 33 ambulant patients with positive PCR test and mild symptoms of infection. Exclusion criteria were: (a) age $>65$ years old, (b) reduced LVEF $(<50 \%)$ at baseline echo, (c) pre-existing cardiovascular or pulmonary disease, (d) atrial and ventricular arrhythmias, (e) renal failure ( $<30 \mathrm{ml} / \mathrm{min}$ ), (f) LV hypertrophy, (g) suboptimal image quality, and (h) unwillingness of the patient to participate. Patients' medical history and laboratory findings were retrospectively collected from medical records. Upon their admission, a complete blood cell analysis and blood biochemistry was performed, including kidney function, electrolytes, C-reactive protein, D-Dimers and hs-cTnI.

Thirtyseven healthy volunteers of similar age and clinical characteristics were used as a control group. All the participants were informed about the protocol and signed a consent form. The study protocol was approved by the scientific committees of both hospitals.

\section{D echocardiography}

The echocardiographic evaluation of all patients was performed after a minimum of 30 days from the initial diagnosis for the outpatients and 10 days after their discharge for the hospitalized patients, provided negative swab test for all of them. The echo examination took place in the left lateral decubitus position with a commercially available system (General Electric Vivid 7 Dimension fully updated, Horten, Norway) using a $3.5 \mathrm{MHz}$ (M4S) transducer. All studies were digitally stored using a computerized station (EchoPAC, version 110, GE, Horten, Norway) and were processed offline by the same investigator-cardiologist.

Conventional echocardiographic measurements were made according to the recommendations of the American Society of Echocardiography and the European Association of Cardiovascular Imaging [11]. From the parasternal long axis view Interventricular septum (IVS) thickness, LV end-diastolic diameter, posterior wall thickness and LV endsystolic diameter were measured. The LV systolic function was assessed using two-dimensional LVEF measured from the apical 4 chamber $(4 \mathrm{CH})$ and 2 chamber $(2 \mathrm{CH})$ views using the modified biplane Simpson method. Right ventricular systolic function was estimated from the S' wave derived from the free wall tricuspid annulus using Tissue Doppler Imaging (TDI). Diastolic function was assessed from $4 \mathrm{CH}$ view measuring the $\mathrm{E}$ and $\mathrm{A}$ waves of the transmittal flow using pulse-wave Doppler and $\mathrm{E}^{\prime}$ and $\mathrm{A}^{\prime}$ from TDI according to the latest guidelines [12]. Mean E' represents the mean early diastolic velocity derived from the lateral mitral annulus and basal interventricular septum. E/A and E/mean $\mathrm{E}^{\prime}$ ratios were also calculated.

\section{D-speckle tracking}

Peak systolic left ventricular global longitudinal strain (LVGLS) was calculated offline by the same investigator using a dedicated software package (EchoPAC, version 110 , Horten, Norway) according to current recommendations [11]. Frame rates of 60-80 frames/sec were used, after optimal sector width and depth adjustments.

LVGLS was measured using the Automated Function Imaging protocol $[13,14]$ for Bull's eye projection as the mean of the 17 ventricular segments from the $4 \mathrm{CH}-, 2 \mathrm{CH}-$, and $3 \mathrm{CH}$-apical views. $\mathrm{LV}$ endocardial borders were manually indicated by 3 points over one frame and then they were automatically tracked throughout the cardiac cycle. A region of interest (ROI) was then presented by the software. The adequacy of tracking and the ROI were manually verified. $L V$ was divided into six equal segments by the 
software, which generated myocardial strain curves. From these curves, peak-systolic strain values were recorded for each myocardial segment. Three consecutive cardiac cycles of each view were acquired and systolic timing was clarified by the aortic valve closure, from the apical long-axis view. Cut-off value for abnormal LVGLS was $<18$ (absolute value) [15]. Regional strains of all LV walls were separately calculated as the mean strain value of the three segments (basal, medial and apical) of each myocardial wall. Finally, right ventricular global longitudinal strain (RVGLS) was also assessed, measuring only the RV free wall strain, and not the IVS strain [16], from a focused-on- $\mathrm{RV}$ modified $4 \mathrm{CH}$ view. All strain measurements referred in the text are absolute values.

\section{Investigator variability}

All measurements were performed by the same investigator. To assess intraobserver variability, the investigator repeated the measurements in twenty of the patients. The intraobserver variability was calculated as the standard deviation (SD) of the differences between the first and second measurements and expressed as a percentage of the average value. Values of intra-observer variability were $4.3 \%$ for LVGLS, $5 \%$ for RVGLS, $5.5 \%$ for LATLS, $5.7 \%$ for POSTLS, $6.1 \%$ for IVSLS, $6.5 \%$ for ANTLS, 5.7\% for INFLS.

\section{Statistical analysis}

All statistical analyses were performed using the Statistical Package for the Social Sciences software (version 20.0.0, IBM SPSS Inc, Chicago, IL, USA). Results are expressed as mean values \pm standard deviation (SD) for the continuous variables and as absolute (N) and relative (\%) frequencies for the categorical ones. Normal distribution of the echocardiographic indices was checked with Kolmogorov-Smirnov and Shapiro-Wilk tests Confirmation of distributions normality was followed by estimating the overall differences between subgroups exerting one-way ANOVA test. Student's t-test and Pearson's chi-square tests were also performed for between-group comparisons. A p-value $<0.05$ was considered statistically significant.

\section{Results}

The baseline characteristics of our cohort are presented in Table 1. Of the 100 patients enrolled, 67 were hospitalized and 33 were treated ambulant. The mean time of performed echo examination of all patients was $33.28 \pm 9.4$ days after the initial confirmed Covid-19 infection diagnosis (for non-hospitalized $42.72 \pm 9$, for hospitalized 28.82 $\pm 5.6 \mathrm{p}<0.0001)$. Hypertension, hyperlipidemia and DM were significantly more prevalent in controls compared to COVID-19 group $(64.9 \%, 81.1 . \%$ and $86.5 \%$ vs. $39.4 \%$, $36.4 \%$ and $59.6 \%, \mathrm{p}=0.008,<0,001$ and 0.003 respectively) (Table 1). Gender and smoking distribution did not differ significantly between the two study groups (Table 1).

Descriptive echocardiographic indices of all groups are presented in Table 2. As far as conventional echocardiographic parameters are concerned, no significant difference was noticed between patients and controls, including LVEF $(59.22 \pm 3.72$ vs. $60.14 \pm 3.19 \%$ respectively, Table 2 ; Fig. 1). LVGLS was found to be significantly lower in Covid-19 recovered patients compared to controls (18.47 \pm 2.4 vs. $21.07 \pm 1.76 \%$ respectively, $p<0.0001$, Table 2 ; Figs. 1 and 2). Analysis of the subgroup of mildly sick non-hospitalized patients revealed that although LVEF did not differ neither from controls $(59.15 \pm 3.76$ vs. 60.14 $\pm 3.19 \%$ respectively), nor from hospitalized Covid-19 patients $(59.15 \pm 3.76$ vs. $59.25 \pm 3.73 \%$ respectively $)$, LVGLS was significantly higher than hospitalized patients $(19.98 \pm 2.92$ vs. $17.72 \pm 1.68 \%$ respectively, $\mathrm{p}<0.0001$, Table 2; Figs. 1 and 2). Compared to controls, non-hospitalized patients showed a trend towards significantly reduced LVGLS $(19.97 \pm 2.91$ vs. $21.07 \pm 1.76 \%$ respectively, $\mathrm{p}=0.068$, Fig. 2). Regional strain analysis revealed that only the lateral wall longitudinal strain (LATLS) and posterior wall longitudinal strain (POSTLS) were significantly reduced in all patients compared to controls $(17.77 \pm 3.48$ vs. $20.96 \pm 2.86 \%$ respectively, $\mathrm{p}<0.0001$ for LATLS and $19.52 \pm 5.3$ vs. $22.23 \pm 2.65 \%$ respectively, $\mathrm{p}=0.01$ for POSTLS, Figs. 1 and 2), while no significant difference was found in IVS, anterior and inferior walls (Fig. 2; Table 2). No difference was found in LATLS and POSTLS between hospitalized and non-hospitalized patients with Covid-19 (Fig. 1; Table 2), while, only LATLS of non-hospitalized patients was lower compared to controls $(18.59 \pm 4.56$ vs. $20.97 \pm 2.86 \%$ respectively, $\mathrm{p}=0.01)$. Finally, RVGLS was found significantly diminished only in the hospitalized group compared to controls $(18.17 \pm 3.32$ vs. $26.03 \pm 4.55 \%$ respectively, p $<0.0001$, Fig. 3), while non-hospitalized patients did not differ significantly from the control group $(27.32 \pm 5.58$ vs. $26.03 \pm 4.55 \%$ respectively, $p=0.076)$. After evaluating the potential confounding effect of hypertension, hyperlipidemia and DM as their distribution differed between the two study groups (COVID-19 and controls), multivariate analysis of variance was performed, adjusting for the aforementioned co-variates (Table 3). According to the multivariate analysis, LVGLS, LATLS, POSTLS and RVGLS were significantly lower in the Covid-19 group compared to controls, even after adjusting for the potential confounding effect of hypertension, hyperlipidemia and DM.

As far as laboratory parameters are concerned, both hs-cTnI and D-Dimers in Covid-19 patients were within normal limits. Also, no correlation was found between 
Table 1 Baseline patient characteristics and comparison between groups

\begin{tabular}{|c|c|c|c|c|c|c|c|c|c|}
\hline & & Group & & & & & & & \\
\hline & & Covid- & & & & & Con & & \\
\hline & & $N$ & & $\%$ & & $p$ & $\bar{N}$ & $\%$ & $p$ \\
\hline & & Hosp & N-Hosp & Hosp & N-Hosp & & & & \\
\hline Gender & Men & 51 & & $51.0 \%$ & & & 14 & 37.8 & 0.171 \\
\hline & & 38 & 13 & 56.7 & 39.4 & 0.103 & & & \\
\hline & Women & 49 & & $49.0 \%$ & & & 23 & 62.2 & \\
\hline & & 29 & 20 & 43.3 & 60.6 & & & & \\
\hline HT & Yes & 39 & & $39.4 \%$ & & & 24 & 64.9 & 0.008 \\
\hline & & 11 & 28 & 16.4 & 87.5 & $<0.001$ & & & \\
\hline & No & 60 & & $60.6 \%$ & & & 13 & 35.1 & \\
\hline & & 56 & 5 & 83.6 & 12.5 & & & & \\
\hline Current Smoking & Yes & 70 & & $70.7 \%$ & & & 26 & 70.3 & 0.960 \\
\hline & & 42 & 28 & 62.7 & 87.5 & 0.011 & & & \\
\hline & No & 29 & & $29.3 \%$ & & & 11 & 29.7 & \\
\hline & & 25 & 5 & 37.3 & 12.5 & & & & \\
\hline Hyperlipidemia & Yes & 36 & & $36.4 \%$ & & & 30 & 81.1 & $<0.001$ \\
\hline & & 6 & 30 & 9 & 93.8 & $<0.001$ & & & \\
\hline & No & 63 & & $63.6 \%$ & & & 7 & 18.9 & \\
\hline & & 61 & 3 & 91 & 6.2 & & & & \\
\hline $\mathrm{DM}$ & Yes & 59 & & $59.6 \%$ & & & 32 & 86.5 & 0.003 \\
\hline & & 29 & 30 & 43.3 & 93.8 & $<0.001$ & & & \\
\hline & No & 40 & & $40.4 \%$ & & & 5 & 13.5 & \\
\hline & & 38 & 3 & 56.7 & 6.2 & & & & \\
\hline & Mean & & SD & & & $\mathrm{p}$ & Mean & SD & $\mathrm{p}$ \\
\hline & Hosp & N-Hosp & Hosp & & & & & & \\
\hline Age(years) & 47.2 & & 9.1 & & & & 46.9 & 6.3 & 0.815 \\
\hline & 49.4 & 42.8 & 9.3 & 6.9 & & 0.001 & & & \\
\hline
\end{tabular}

hs-cTnI and D-Dimers values and reduction of LVGLS and RVGLS.

\section{Discussion}

The main findings of our study are:

(1) LVGLS was significantly impaired in Covid-19 recovered patients compared to controls. The reduction of LVGLS was found to concern specifically the posterolateral wall.

(2) LVGLS impairment was found to be independent of the clinical manifestation and the severity of Covid-19 infection and was reduced in both groups of patients, those with pneumonia and severe respiratory insufficiency and those who were treated ambulant.

(3) RVGLS was particularly reduced in hospitalized Covid19 patients and was correlated to disease severity.
(4) No correlation was found between GLS values and biomarker levels especially hs-cTnI and D-Dimers.

GLS is a very sensitive echocardiographic marker that reveals subclinical myocardial dysfunction, even in the cases of an apparently normal ventricular pump function based upon normal EF. There are a few studies in the literature that reveal cardiac involvement using GLS in patients recovered from Covid 19 infection [17-19]. In accordance to these studies, we showed that LVGLS is impaired in 50\% of Covid-19 patients. Moreover, in accordance with other published studies $[19,20]$, we found that disease severity does not influence the extend of LVGLS impairment. Although one study [21] demonstrated significantly lower LVGLS values after discharge only in the group with severe pneumonia, the fact that the study's cut-off value of impaired GLS was $<16$ (absolute values), may be the cause of this discrepancy. Consequently, it seems reasonable to conclude that patients who have recovered from Covid 19 might be 


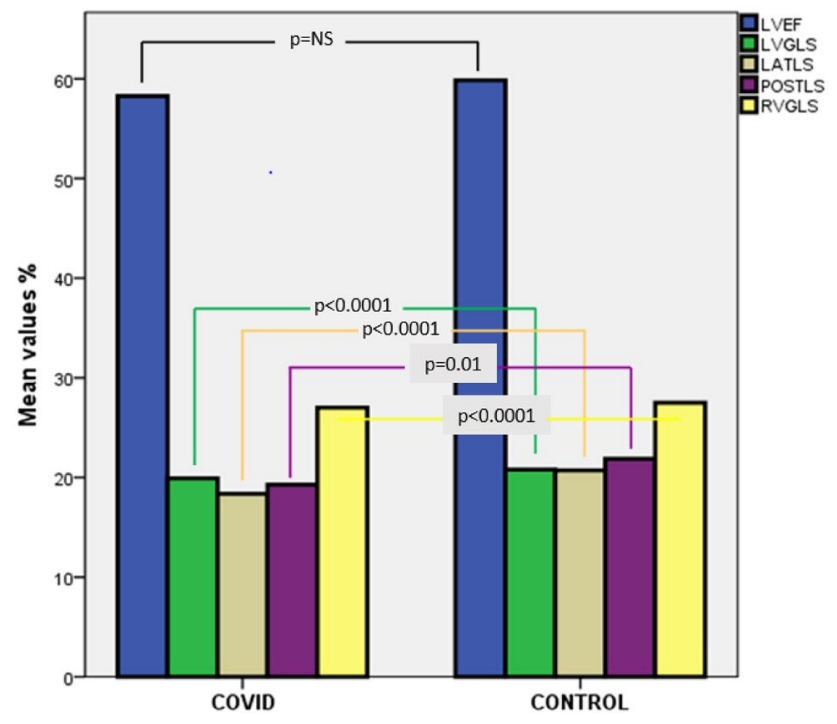

Fig. 1 Schematic representation of LVEF, LVGLS, LATLS, POSTLS and RVGLS of recovered Covid-19 patients compared to controls. $L V E F$ left ventricular ejection fraction, $L V G L S$ left ventricular global longitudinal strain, LATLS lateral wall longitudinal strain, POSTLS posterior wall longitudinal strain, RVGLS right ventricular global longitudinal strain. at risk of myocardial injury regardless of the severity of the respiratory symptoms. This injury has been found to be in the spectrum of subclinical dysfunction and further studies are needed in order to confirm the benign, reversible, uncomplicated evolution or not, of this observation.

In addition to that, our study demonstrated particularly impaired longitudinal strain mainly of the LV lateral wall but also, in a lesser extent, of the posterior wall in all patients with Covid-19 infection. Previous studies have reported that in patients with myocarditis and preserved EF, reduced LATLS is well correlated with Magnetic Resonance Imaging (MRI) finding of late gadolinium enhancement (LGE) in the postero-lateral myocardial wall [22]. In other words, based upon these findings, a logical conclusion could be that patients who experience Covid-19 infection demonstrate myocardial inflammation similar to that of viral myocarditis. Finally, another important finding of our study is that while no difference was found in LATLS and POSTLS between hospitalized and non-hospitalized patients with Covid-19, only LATLS of non-hospitalized patients was reduced compared to controls, highlighting LATLS as the most sensitive indicator of cardiac injury after virus infection. As it has
Fig. 2 Longitudinal strain Bull's eye plot in A non-hospitalized and $\mathbf{B}$ hospitalized Covid-19 patients. In both groups LVGLS was reduced, more seriously in hospitalized patients and mainly in the lateral and posterior wall. C. LVEF was normal and similar in both groups of patients. $L V G L S$ left-ventricular global longitudinal strain, $L V E F$ leftventricular ejection fraction, GLPS global longitudinal peak systolic strain, $L A X 3$ chamber view, $A 4 C 4$ chamber view, $A 2 C 2$ chamber view, $A v g$ average
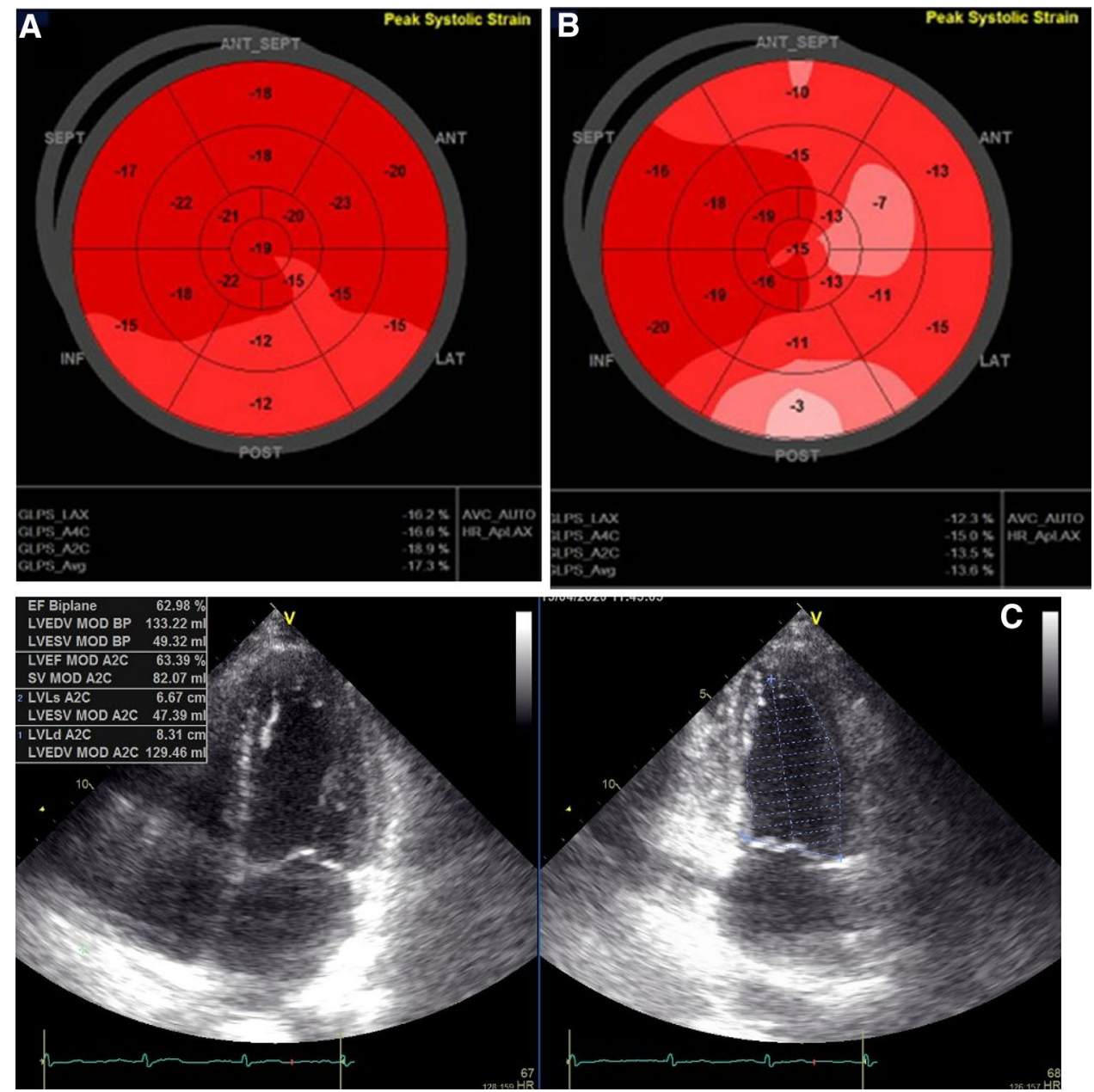
Fig. 3 RVGLS in A Hospitalized and $\mathbf{B}$ non-hospitalized Covid-19 patients. RVGLS rightventricular global longitudinal strain
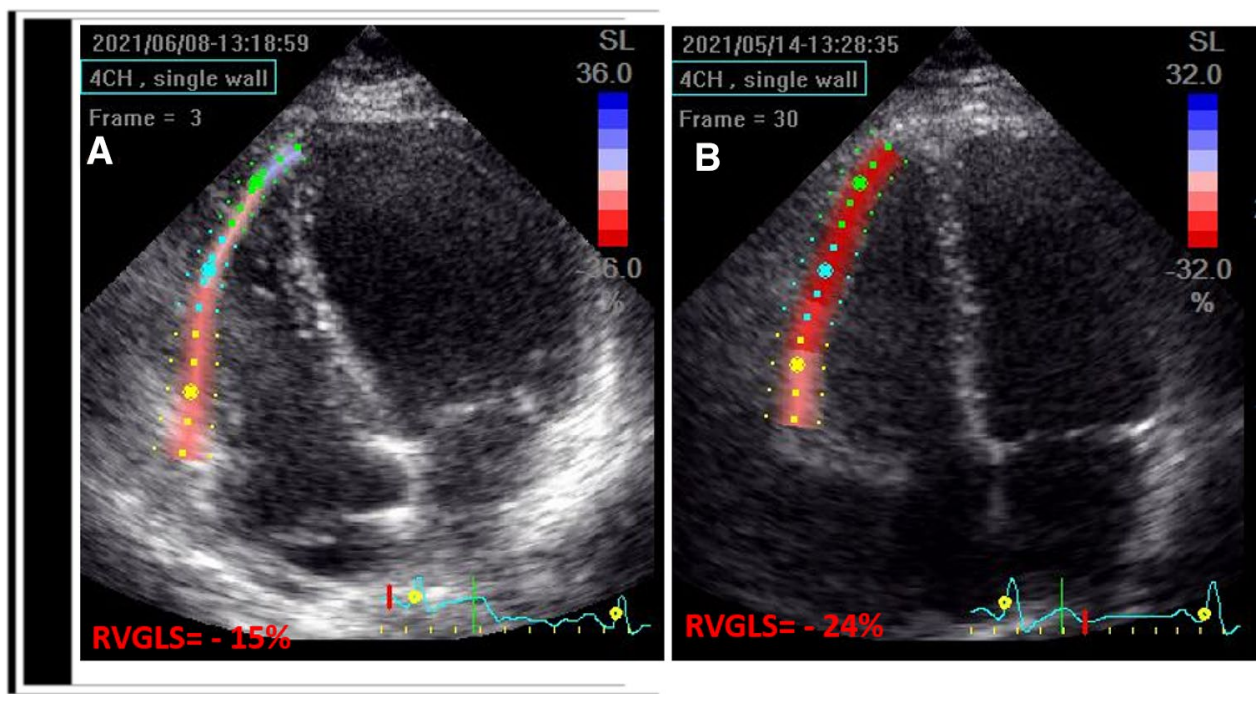

Table 2 Conventional and strain echocardiographic parameters of hospitalized, non-hospitalized Covid-19 patients and controls

\begin{tabular}{|c|c|c|c|c|c|}
\hline & Covid Hosp. & Covid N hosp. & Controls & $\begin{array}{l}\text { P } \\
\text { Hosp vs. N-hosp }\end{array}$ & $\begin{array}{l}\mathrm{p} \\
\text { Controls vs. Covid }\end{array}$ \\
\hline $\mathrm{N}$ & 67 & 33 & 37 & & \\
\hline IVS (mm) & $8.67 \pm 1.06$ & $7.62 \pm 1.19$ & $8.28 \pm 0.75$ & 0.068 & 0.847 \\
\hline PW (mm) & $8.76 \pm 1.05$ & $7.47 \pm 1.09$ & $8.09 \pm 0.91$ & 0.107 & 0.271 \\
\hline LVEDD (mm) & $46.55 \pm 4.71$ & $46.91 \pm 2.95$ & $47.22 \pm 4.13$ & 0.692 & 0.499 \\
\hline LVESD (mm) & $29.34 \pm 5.53$ & $30.58 \pm 2.48$ & $30.24 \pm 3.68$ & 0.227 & 0.571 \\
\hline $\mathrm{E} / \mathrm{A}$ & $1.08 \pm 0.39$ & $1.29 \pm 0.43$ & $1.26 \pm 0.30$ & 0.017 & 0.136 \\
\hline $\mathrm{E} / \mathrm{E}$ & $7.44 \pm 2.22$ & $7.13 \pm 1.79$ & $7.26 \pm 2.12$ & 0.465 & 0.858 \\
\hline $\mathrm{S}(\mathrm{m} / \mathrm{sec})$ & $14.18 \pm 2.66$ & $13.47 \pm 2.21$ & $13.07 \pm 1.81$ & 0.192 & 0.062 \\
\hline LVEF (\%) & $59.25 \pm 3.73$ & $59.15 \pm 3.76$ & $60.14 \pm 3.19$ & 0.898 & 0.187 \\
\hline $\operatorname{AVmax}(\mathrm{m} / \mathrm{s})$ & $1.23 \pm 0.22$ & $1.28 \pm 0.16$ & $1.29 \pm 0.16$ & 0.205 & 0.219 \\
\hline LVGLS (\%) & $-17.72 \pm-1.68$ & $-19.97 \pm-2.91$ & $-21.07 \pm-1.75$ & $<0.0001$ & $<0.0001$ \\
\hline LATLS (\%) & $-17.38 \pm-2.79$ & $-18.59 \pm-4.56$ & $-20.96 \pm-2.86$ & 0.109 & $<0.001$ \\
\hline POSTLS (\%) & $-19.52 \pm-5.30$ & $-18.90 \pm-2.26$ & $-22.23 \pm-2.65$ & 0.12 & 0.01 \\
\hline RVGLS (\%) & $-18.17 \pm-3.32$ & $-27.32 \pm-5.58$ & $-26.03 \pm-4.55$ & $<0.001$ & $<0.001$ \\
\hline
\end{tabular}

IVS interventricular septum, $P W$ posterior wall, $L V E D D$ left ventricular end-diastolic diameter, $L V E S D$ left ventricular end-systolic diameter, $L V E F$ left ventricular ejection fraction, $L V G L S$ left ventricular global longitudinal strain, $L A T L S$ longitudinal strain of lateral wall, POSTLS longitudinal strain of posterior wall, RVGLS right ventricular global longitudinal strain

already been referred, Kostakou et al. demonstrated that reduced LATLS could be a predictive tool of myocarditis in patients with preserved ejection fraction [22] and many other studies have connected reduced strain values to myocarditis [23-25]. Moreover, Puntman et al. evaluated the presence of myocardial injury in 100 patients recently recovered from Covid 19 infection using MRI and revealed cardiac involvement in $78 \%$ of the patients while confirming myocardial inflammation in $60 \%$ of them [9].

The myocardial dysfunction caused in hospitalized patients with pneumonia caused by Covid-19 seems to be multifactorial. One mechanism of the myocardial damage could be direct myocardium injury through virus binding to angiotensin-converting enzyme 2 receptors or indirect infiltration with cytokines and chemokines, in other words myocarditis [25]. The latter mechanism consists of a systematic inflammatory response to Covid-19 infection, already known as cytokine storm, where endothelium may be displayed to proinflammatory cytokines, promoting molecule and chemokine attachment that leads to leukocyte aggregation and inflammation [26]. A third mechanism could be the over-activation of autoimmune system with increased interferon effluence causing extreme implementation of innate and adaptive immune biological pathways [26]. On the other hand, hypoxemia, right ventricular strain and thrombotic complications could also be potential causes of the 
Table 3 Multivariate analysis of variance for each variable of interest

\begin{tabular}{|c|c|c|c|c|c|}
\hline & \multicolumn{4}{|l|}{ Group } & \multirow[t]{3}{*}{$\mathrm{p}$} \\
\hline & \multicolumn{2}{|c|}{ Covid-19 } & \multicolumn{2}{|c|}{ Controls } & \\
\hline & Mean & SD & Mean & $\mathrm{SD}$ & \\
\hline LVEF & 59.22 & 3.72 & 60.14 & 3.20 & 0.187 \\
\hline LVGLS & 18.47 & 2.40 & 21.07 & 1.76 & $<0.001$ \\
\hline LATLS & 17.77 & 3.49 & 20.97 & 2.86 & $<0.001$ \\
\hline POSTLS & 19.52 & 5.30 & 22.23 & 2.65 & 0.020 \\
\hline RVGLS & 21.29 & 5.88 & 26.04 & 4.55 & $<0.001$ \\
\hline IVSLS & 20.11 & 3.58 & 20.12 & 2.62 & 0.988 \\
\hline ANTLS & 20.70 & 2.19 & 21.60 & 2.84 & 0.437 \\
\hline INFLS & 20.58 & 4.11 & 21.76 & 2.56 & 0.192 \\
\hline
\end{tabular}

All analyses were performed adjusting for the effect of hypertension, hyperlipidemia and DM. $L V E F$ left ventricular ejection fraction, $L V G L S$ left ventricular global longitudinal strain, $L A T L S$ longitudinal strain of lateral wall, POSTLS longitudinal strain of posterior wall, $R V G L S$ right ventricular global longitudinal strain, IVSLS longitudinal strain of interventricular septum, ANTLS longitudinal strain of anterior wall, INFLS longitudinal strain of inferior wall

myocardial dysfunction [25]. Histological evidence, from biopsy, of myocardial injury or myocarditis by Covid-19, is limited and controversial, as it is not clarified whether Covid-19 impairs directly the heart or not [27]. Our finding of regional impaired strain suggests a direct infection of myocardium by coronavirus rather than myocardial injury secondary to systemic causes.

Another important finding of our study was that subclinical myocardial dysfunction regarded not only the left but also the right ventricle. More specifically, impaired RVGLS was found in 51\% of Covid-19 patients who were hospitalized for Covid-19 pneumonia, while non-hospitalized patients had a normal RVGLS value. Li et al. reported that RVGLS had a trend towards impairment in the severe group of Covid-19 patients, although not statistically significant [28]. RVGLS can be a prognostic parameter of severe disease, as suggested from a previous study, which confirmed that RVGLS was related to clinical outcome in 35 patients with Covid-19 pneumonia [29]. This finding is of great importance, because in that way, patients who require intensive care might be detected earlier. Although in some cases RVGLS deterioration can be the consequence of LV dysfunction, in our study the LVEF of all patients was in normal range. All of the above make imperative the necessity of more comprehensive approach and evaluation of the RVGLS role in Covid-19 patients.

Troponin is a very sensitive biomarker, which can detect myocardial damage in a variety of clinical situations. According to the previously edited literature, troponin is considered to be an independent predictor of adverse clinical outcomes in Covid-19 patients. Bieber et al. proposed a diagnostic pathway of early identification of myocardial damage by biomarkers and subsequently revealing and characterizing by comprehensive three- dimensional STE the extent and pattern of myocardial dysfunction in Covid19 infected patients [30]. On the other hand Kocas et al., who categorized their Covid-19 infected population of 38 subjects in a positive and a negative troponin group, did not find any significant LVGLS difference between the groups which is consistent with our study. Taking into consideration that according to Smith et al. cTnI is elevated only in $34 \%$ of biopsy- proven myocarditis [31], speckle tracking echocardiography could be a much more sensitive technique for detecting subclinical myocardial damage.

Although many studies have revealed subclinical myocardial dysfunction induced from coronavirus infection, the significance of our study lies in the fact that the myocardial dysfunction continued after the acute phase of the disease, once the respiratory symptoms were resolved., which is in accordance with the previously mentioned MRI study [9] which reported that cardiac involvement has been found to persist beyond the period of acute presentation, and creates concerns about the long-term cardiovascular complications of Covid-19. The ongoing myocardial damage may imply that Covid-19 patients are highly susceptible to the development of heart failure in the future. Due to the fact that Covid19 is an emerging threat for the heart and the early detection of residual myocardial complications in Covid-19 survivors is very important, the routine use of echocardiography and strain analysis in recovered Covid-19 patients could be considered as highly recommended.

Limitations

(1) RV strain analysis was performed using the LV specific strain software, due to unavailability of RV specific software in our lab. However, these two methods correlate very well even if they are not totally identical.

(2) Obese patients, who constitute a considerable portion of individuals affected by Covid-19, create an extra difficulty in the effort of image acquisition.

(3) Our cohort comprised only adults after the acute infection phase, so the findings were not validated for patients younger than 18 years old and they also do not represent the acute or asymptomatic phase of Covid-19 infection.

\section{Conclusions}

Covid-19 is a new multisystem disease that it seems to affect LV myocardium independently of disease severity, while its consequences could be extended, meaning that recovered patients have to be monitored for a long time. This article highlights the need for long-term follow-up of patients who experienced Covid-19 infection, as LV is affected almost 
in all individuals with LATLS being the most sensitive marker of LV impairment. Furthermore, in our study RV shows signs of dysfunction only in severely ill patients highlighting RVGLS as a helpful tool of prognosis. In order to face the emergency for effective treatment and prevention of long Covid-disease, an orchestrated effort should be made for comprehensive interpretation of biological mechanisms and clinical signs of coronavirus infection as soon as possible. Potentially, this process will lead to earlier detection and implementation of cardioprotective treatment strategies.

Author contributions All authors included in this work have (1) made substantial contributions to the conception or design of the work; or the acquisition, analysis, or interpretation of data; or the creation of new software used in the work; (2) drafted the work or revised it critically for important intellectual content; (3) approved the version to be published; and (4) agree to be accountable for all aspects of the work in ensuring that questions related to the accuracy or integrity of any part of the work are appropriately investigated and resolved.

Funding This research did not receive any specific grant from funding agencies in the public, commercial, or not-for-profit sectors.

Data availability All studies were digitally stored using a computerized station (EchoPAC, version 110, GE, Horten, Norway). All the measurements have been stored in an XL MS Office document.

Code Availability Every patient has been decoded using a number from 1 to 100 and only this code number is used when processing the data.

\section{Declarations}

Conflict of interest The authors declare that they have no conflict of interest.

Ethical approval The study has been approved by the scientific committee of Thriassio General Hospital and Korinthos General Hospital and has therefore been performed in accordance with the ethical standards laid down in the 1964 Declaration of Helsinki and its later amendments.

Consent to participate/consent for publication All participants have signed an informed consent.

\section{References}

1. Evans P, Rainger G, Mason J, Guzik T, Osto, Stamataki Z et al (2020) Endothelial dysfunction in COVID-19: a position paper of the ESC Working Group for Atherosclerosis and Vascular Biology, and the ESC Council of Basic Cardiovascular Science. Cardiovasc Res 116:2177-2184

2. Shi S, Qin M, Shen B, Cai Y, Liu T, Yang F et al (2020) Association of cardiac injury with mortality in hospitalized patients with COVID-19 in Wuhan, China. JAMA Cardiol 5:802-810

3. Ruan Q, Yang K, Wang W, Jiang L, Song J (2020) Clinical predictors of mortality due to COVID-19 based on an analysis of data of 150 patients from Wuhan, China. Intensive Care Med 46:846-848
4. Zhou F, Yu T, Du R, Fan G, Liu Y, Liu Z (2020) Clinical course and risk factors for mortality of adult inpatients with COVID19 in Wuhan, China: a retrospective cohort study. Lancet 395:1054-1062

5. Riphagen S, Gomez X, Gonzalez-Martinez C, Wilkinson N, Theocharis P (2020) Hyperinflammatory shock in children during COVID-19 pandemic. Lancet 395:1607-1608

6. Huang C, Wang Y, Li X, Ren L, Zhao J, Hu Y et al (2020) Clinical features of patients infected with 2019 novel coronavirus in Wuhan, China. Lancet 6736:1-10

7. Wang D, Hu B, Hu C, Zhu F, Liu X, Zhang J et al (2020) Clinical characteristics of 138 hospitalized patients with 2019 Novel Coronavirus-infected pneumonia in Wuhan, China. JAMA 323:1601-1609

8. Wang F, Kream R, Stefano G (2020) Long-term respiratory and neurological sequelae of COVID-19. Med Sci Monit 26:e928996

9. Puntmann V, Carerj M, Wieters I, Fahim M, Arendt C, Hoffmann J et al (2020) Outcomes of cardiovascular magnetic resonance imaging in patients recently recovered from coronavirus disease 2019 (COVID-19). JAMA Cardiol 5:1265-1273

10. Huang L, Zhao P, Tang D, Zhu T, Han R, Zhan C et al (2020) Cardiac involvement in patients recovered from COVID-2019 identified using magnetic resonance imaging. JACC Cardiovasc Imaging 13:2330-2339

11. Lang RM, Badano LP, Mor-Avi V, Afilalo J, Armstrong A, Ernande L et al (2015) Recommendations for cardiac chamber quantification by echocardiography in adults: an update from the American Society of Echocardiography and the European Association of Cardiovascular Imaging. J Am Soc Echocardiogr 28:1-39

12. Nagueh S, Smiseth O, Appleton C, Byrd B, Dokainish H, Edvardsen $\mathrm{T}$ et al (2016) Recommendations for the evaluation of left ventricular diastolic function by echocardiography: an update from the american society of echocardiography and the european association of cardiovascular imaging. Eur Heart J Cardiovasc Imaging 17:1321-1360

13. Barbier P, Mirea $O$, Cefalù C, Maltagliati A, Savioli G, Guglielmo M (2015) Reliability and feasibility of longitudinal AFI global and segmental strain compared with $2 \mathrm{D}$ left ventricular volumes and ejection fraction: intra- and inter-operator, test-retest, and intercycle reproducibility. Eur Heart J Cardiovasc Imaging 16:642-652

14. Anwar A (2012) Global and segmental myocardial deformation by $2 \mathrm{D}$ speckle tracking compared to visual assessment. World $\mathrm{J}$ Cardiol 4:341-346

15. Kocabay G, Muraru D, Peluso D, Cucchini U, Mihaila S, Padayattil-Jose $S$ et al (2014) Normal left ventricular mechanics by twodimensional speckle-tracking echocardiography. Reference values in healthy adults. Rev Esp Cardiol 67:651-658

16. Badano L, Kolias TJ, Muraru D, Abraham T, Aurigemma G, Edvardsen T et al (2018) Standardization of left atrial, right ventricular, and right atrial deformation imaging using two dimensional speckle tracking echocardiography: a consensus document of the EACVI/ASE/Industry Task Force to standardize deformation imaging. Eur Heart J Cardiovasc Imaging 19:591-500

17. Özer S, Candan L, Özyıldız AG, Turan OE (2021) Evaluation of left ventricular global functions with speckle tracking echocardiography in patients recovered from COVID-19. Int J Cardiovasc Imaging 37:2227-2233

18. Li R, Wang H, Ma F, Cui G, Peng L, Li C et al (2021) Widespread myocardial dysfunction in COVID-19 patients detected by myocardial strain imaging using 2-D speckle-tracking echocardiography. Acta Pharmacol Sin 42(10):1567-1574

19. Croft L, Krishnamoorthy P, Ro R, Anastasius M, Zhao W, Buckley $S$ et al (2021) Abnormal left ventricular global longitudinal strain by speckle tracking echocardiography in COVID-19 patients. Future Cardiol 17:655-661 
20. Baycan O, Barman H, Atici A, Tatlisu A, Bolen F, Ergen P et al (2021) Evaluation of biventricular function in patients with COVID19 using speckle tracking echocardiography. Int J Cardiovasc Imaging 37:135-144

21. Baykiz D, Govdeli EA, Ozer PK, Karaayvaz EB, Catma Y, Medetalibeyoglu A (2021) Evaluation the relationship of left ventricular global longitudinal strain and laboratory parameters in discharged patients with COVID-19: a follow-up study. Int J Cardiovasc Imaging 37:2451-2464

22. Kostakou P, Kostopoulos V, Tryfou E, Giannaris V, Rodis I, Olympios C et al (2018) Subclinical left ventricular dysfunction and correlation with regional strain analysis in myocarditis with normal ejection fraction. A new diagnostic criterion. Int J Cardiol 259:116-121

23. Gursu H, Cetin I, Azak E, Kibar A, Surucu M, Orgun A et al (2019) The assessment of treatment outcomes in patients with acute viral myocarditis by speckle tracking and tissue Doppler methods. Echocardiography 36:1666-1674

24. Uppu S, Shah A, Weigand J, Nielsen J, Ko H, Parness I et al (2015) Two-dimensional speckle-tracking-derived segmental peak systolic longitudinal strain identifies regional myocardial involvement in patients with myocarditis and normal global left ventricular systolic function. Pediatr Cardiol 36:950-959

25. Basso C, Leone O, Rizzo S, Gaspari M, Wal A, Aubry MC (2020) Pathological features of COVID-19-associated myocardial injury: a multicentre cardiovascular pathology study. Eur Heart J 41:3827-3835
26. Chousterman B, Swirski F, Weber G (2017) Cytokine storm and sepsis disease pathogenesis. Semin Immunopathol 39:517-528

27. Nishiga M, Wang D, Han Y, Lewis D, Wu J (2020) COVID-19 and cardiovascular disease: from basic mechanisms to clinical perspectives. Nat Rev Cardiol 17:543-558

28. Kim M, Nam J, Son J, Kim S, NH S, Ahn C et al (2020) Cardiac manifestations of coronavirus disease 2019 (COVID-19): a multicenter cohort study. J Korean Med Sci 35:e366

29. Stockenhuber A, Vrettos A, Androschuck V, George M, Robertson C, Bowers N et al (2021) A pilot study on right ventricular longitudinal strain as a predictor of outcome in COVID-19 patients with evidence of cardiac involvement. Echocardiography 38:222-229

30. Bieber S, Kraechan A, Hellmuth J, Muenchhoff M, Scherer C, Schroeder I et al (2021) Left and right ventricular dysfunction in patients with COVID-19-associated myocardial injury. Infection 49:491-400

31. Smith S, Ladenson J, Mason J, Jaffe A (1997) Elevations of cardiac troponin I associated with myocarditis: experimental and clinical correlates. Circulation 95:163-168

Publisher's Note Springer Nature remains neutral with regard to jurisdictional claims in published maps and institutional affiliations. 